\title{
ATUAÇÃO DO PSICÓLOGO COM ADOLESCENTES EM CONFLITO COM A LEI: A EXPERIÊNCIA DO CANADÁ ${ }^{1}$
}

Alex Eduardo Gallo*

\begin{abstract}
RESUMO. O presente artigo apresenta o relato de experiência da atuação do psicólogo no sistema de justiça canadense, especialmente no caso de adolescentes em conflito com a lei. É apresentada uma comparação entre o Estatuto da Criança e do Adolescente e leis semelhantes do Canadá, intituladas Youth Criminal Justice Act. São apresentados os procedimentos de avaliação biopsicossocial do adolescente, assim como a execução das medidas socioeducativas chamadas medidas alternativas. Embora a execução das medidas alternativas seja parecida entre os dois países, os índices de violência no Canadá são expressivamente menores do que no Brasil, o que evidencia que não são as medidas de caráter judicial que, isoladamente, reduzem a violência, mas uma série de fatores sociopolíticos, educacionais e culturais envolvidos.
\end{abstract}

Palavras-chave: adolescente em conflito com a lei, violência, educação.

\section{PSYCHOLOGIST' WORK WITH YOUNG OFFENDERS: THE EXPERIENCE OF CANADA}

\begin{abstract}
Current research presents an experience report of a psychologist working within the Canadian justice system with young offenders. A comparison between the Brazilian Child and Adolescent Statute and Canadian similar laws, called Youth Criminal Justice Act is provided. Procedures for the adolescent's bio-psycho-social evaluation, as execution of alternative measures, are also given. Although the execution of alternative measures are similar to those in Brazil, the violence rate in Canada is expressively lower, which demonstrates that merely justice measures do not reduce violence, but socialpolitical, educational and cultural factors are involved too.
\end{abstract}

Key words: Young offenders, violence, education.

\section{EL TRABAJO DEL PSICÓLOGO CON ADOLESCENTES EN CONFLICTO CON LA LEY: LA EXPERIENCIA DEL CANADÁ}

RESUMEN. El presente artículo expone el relato de una experiencia de actuación del Psicólogo en el sistema judicial canadiense, especialmente en casos de adolescentes en conflicto con la Ley. Es presentada una comparación entre el "Estatuto da Criança e do Adolescente" y las leyes semejantes en Canadá, tituladas Youth Criminal Justice Act. Se presentan los procedimientos de evaluación bio-psicosocial del adolescente, así como la ejecución de las medidas socioeducativas, llamadas de medidas alternativas. La ejecución de las medidas alternativas, aunque sea parecida con la brasilera, los índices de violencia en Canadá son expresivamente menores que los de Brasil, lo que evidencia que no son solamente las medias de carácter judicial que reducen la violencia, sino una serie de factores sociopolíticos, educacionales y culturales envolvimos.

Palabras-clave: Adolescente en conflicto con la ley, violencia, educación.

A violência, diariamente presente em jornais, tem preocupado a sociedade. Em grande parte das ocorrências registradas pela polícia, os adolescentes figuram como autores, embora os dados de Oliveira e Assis (1999) mostrem que grande parte dos adolescentes são vítimas, principalmente, de morte violenta, o que evidencia não só um problema de segurança pública, mas também de saúde pública e de educação.

Apoio: CNPq.

* Professor do Centro Universitário de Maringá-Cesumar. Pesquisador do Laboratório de Análise e Prevenção da Violência. Universidade Federal de São Carlos. 
Oliveira e Assis (1999) relevam que somente na cidade do Rio de Janeiro a taxa de homicídios praticados por adolescentes de quinze a dezenove anos é cerca de dez vezes maior do que a dos Estados Unidos, onde a taxa de homicídios foi de 10,3 por 100.000 habitantes entre 15 e 19 anos.

Segundo Meneghel, Giugliani e Falceto (1998), o assassinato de adolescentes supostamente delinqüentes pode estar relacionado à violência estrutural, na qual a sociedade lhes nega as condições sociais necessárias. A face repressiva do Estado é quase a única que esses jovens conhecem, sendo que a violência organizada dos grupos de narcotráfico lhes possibilita realizar seus sonhos de afirmação, heroísmo e consumo e obter vantagens imediatas (Meneghel, Giugliani \& Falceto, 1998).

No Canadá os índices de violência são expressivamente menores que os do Brasil. Segundo um levantamento realizado pelo Centro Canadense de Estatísticas da Justiça (Canadian Centre for Justice Statistics), no ano de 1999 a taxa de crimes em geral envolvendo adolescentes foi de 338 por 10.000 adolescentes. Desses crimes, somente $4 \%$ se referiam a delitos contra a pessoa, sendo que a incidência de homicídios foi praticamente nula. Para exemplificar, na cidade de London, no Sudoeste da província de Ontário, com 336.539 habitantes, no ano de 2004 foi registrado somente um homicídio, no geral. Essa estatística não difere substancialmente das demais cidades do país (Canadian Centre for Justice Statistics, 2000).

O presente artigo consiste em narrar a experiência de um psicólogo brasileiro em um centro de referência ao adolescente em conflito com a lei no Canadá, em função de seu processo de doutoramento, especificamente no Center for Children and Families in the Justice System. Esse centro é um órgão independente, mantido com verbas de diferentes Ministérios (recursos para pesquisa, capacitação de profissionais e prestação de serviços técnicos especializados), assim como doações. Esse centro tem como objetivo principal oferecer serviços especializados a crianças, adolescentes e famílias que por algum motivo entraram no sistema de justiça, sendo vítimas, testemunhas, acusados ou defensores. O centro também produz conhecimento, por meio de pesquisas relacionadas a esse tema, assim como oferece capacitação a profissionais ligados ao sistema de justiça.

\section{O SISTEMA DE JUSTIÇA CANADENSE}

O Estatuto da Criança e do Adolescente (Brasil, 1990) considera o jovem até 18 anos como inimputável, dada a sua condição de pessoa em desenvolvimento (Pereira \& Mestriner, 1999). Não havendo tal capacidade, elimina-se a culpabilidade, o que não significa deixar o autor de ato infracional isento de conseqüências, mas submetê-lo às normas da legislação especial. A legislação especial prevê medidas socioeducativas aplicáveis aos adolescentes.

No Canadá há uma lei semelhante ao Estatuto da Criança e do Adolescente: o Youth Criminal Justice Act. Esse conjunto de leis considera o jovem entre 12 e 18 anos como uma pessoa em desenvolvimento, portanto sujeita às normas da legislação especial, mas não considera esse jovem inimputável, dependendo das características do caso. Ou seja, um adolescente que pratica um ato infracional pode ser condenado como se fosse adulto, dependendo de seu histórico anterior em reincidências e da gravidade do ato praticado (Canadian Centre for Justice Statistics, 1998).

Os adolescentes entre 12 e 14 anos de idade que praticarem crime grave, como homicídio, e tenham um histórico de reincidências em crimes contra a pessoa e, repetidamente, hajam sido advertidos pelo cumprimento inadequado de medidas alternativas, podem ser transferidos para o fórum criminal, sendo julgados como adultos. Jovens acima de 16 anos, nesses casos, são automaticamente transferidos, podendo optar pelo julgamento com juiz ou júri popular. As sentenças são, então, as mesmas aplicadas aos adultos, incluindo prisão perpétua. Nesses casos os adolescentes têm direito a liberdade condicional (parole) depois de 10 anos de cumprimento da pena, enquanto os adultos só têm esse direito após 25 anos de cumprimento na prisão (Canadian Centre for Justice Statistics, 1998).

As medidas socioeducativas previstas no Estatuto da Criança e do Adolescente (Brasil, 1990) são semelhantes às medidas alternativas previstas no Youth Criminal Justice Act. A Tabela 1 apresenta as medidas previstas nos dois países.

Tabela 1. Medidas Sócio-Educativas no Brasil e Medidas Alternativas no Canadá.

\begin{tabular}{ll}
\hline Estatuto da Criança e do Adolescente & \multicolumn{1}{c}{ Young Offenders Act } \\
\hline Advertência & Advertência \\
- & Pedido de Desculpas à Vítima \\
- & Multa \\
Obrigação de Reparar o Dano & Obrigação de Reparar o Dano \\
Prestação de Serviços à Comunidade & Prestação de Serviços Comunitários \\
Liberdade Assistida & Probation \\
Semiliberdade & Custódia Aberta \\
Internação & Custódia Fechada \\
\hline
\end{tabular}


A medida de pedido de desculpas à vítima, que não existe na legislação brasileira, pode ser verbal, durante a audiência, ou por escrito, entregue à vítima durante $\mathrm{o}$ julgamento, sempre condicionada a advertência. A multa, que também não tem correspondente no Brasil, é estipulada pelo juiz, em valor sempre acima de 5.000 dólares canadenses. As demais medidas são semelhantes.

O processo judicial canadense é semelhante ao brasileiro. Ele começa com a apreensão do adolescente pela polícia, seja em flagrante, seja após a apuração da investigação. A polícia registra a ocorrência e encaminha o registro para o tribunal, podendo, com base nos indícios de prática de nova infração ou risco à vida, decidir-se pela custódia temporária do adolescente. A autoridade policial tem também o poder de desviar o processo do sistema formal de justiça, em casos que, por não haver severidade, não se requeira julgamento. Por exemplo, se um aluno de 15 anos agredir verbalmente um colega na escola e a direção chamar a polícia, o oficial pode optar por não registrar a queixa da agressão (se a vítima não quiser registrar) e encaminhar $o$ adolescente até seus pais e e com eles discutir as conseqüências de uma futura agressão.

No tribunal, o promotor é responsável pela acusação. Se o adolescente tem recursos financeiros, poderá contratar um advogado de defesa; se não os tem, ele é encaminhado para aconselhamento jurídico, se assim preferir. $\mathrm{O}$ sistema de justiça não prevê a contratação de um defensor pelo Estado. No Brasil, se o acusado não tem como pagar um advogado de defesa, o Estado nomeia um defensor ad hoc, garantindo o princípio de plena defesa. No Canadá o cidadão pode representar a si mesmo no tribunal, não necessitando de um defensor. Aqueles que não podem pagar por um advogado têm à disposição um serviço de aconselhamento jurídico por uma procuradoria (legal-aid).

Tal serviço pode ser prestado por qualquer advogado que exerça sua profissão na cidade, mediante um cadastro no órgão governamental responsável. $\mathrm{O}$ aconselhamento jurídico é definido por uma orientação, feita pelo advogado, para que o cliente tenha conhecimento suficiente para representar a si mesmo no tribunal. O caso é detalhado e é explicado ao cliente como ele deve agir perante o juiz, assim como ele é informado sobre seus direitos legais. Tal serviço pode ser contratado por qualquer pessoa acima de 16 anos, sem a necessidade do consentimento dos pais, e todas as informações são mantidas em sigilo. Os honorários do advogado são pagos pelo governo da província após aprovação dos critérios de elegibilidade para legal-aid.

Para se tornar elegível ao serviço de aconselhamento jurídico, no momento da acusação, o promotor encaminha o adolescente ao setor de legalaid no tribunal. Esse setor verifica as condições econômicas da pessoa. Se o adolescente tiver renda igual ou inferior ao definido, ele recebe a documentação do legal-aid, assim como a lista de advogados da cidade que podem prestar esse serviço. $\mathrm{O}$ adolescente tem o direito de escolher qualquer advogado que aceite esse tipo de serviço.

Após a audiência com o promotor, quando o adolescente é formalmente acusado pelo ato infracional praticado o juiz poderá solicitar maiores informações sobre ele, a fim de determinar a sentença que melhor se lhe aplique, podendo encaminhá-lo para uma avaliação. Isso acontece quando o juizado tem dúvidas sobre se o adolescente de fato tem consciência do delito ou se apresenta indícios de problemas psicológicos. Esta última situação o torna incapaz de participar de um julgamento-padrão.

É muito comum o juiz solicitar uma avaliação psicológica do adolescente no Canadá. Embora essa avaliação esteja prevista para os casos que não se encaixem no padrão, essa norma é subjetiva e depende do ponto de vista das pessoas envolvidas no julgamento. Ela pode ser solicitada pela promotoria ou pela defesa, mas requer a concordância do juiz. Por se tratar de uma regra subjetiva, cada vez mais os juízes têm solicitado uma avaliação, com o objetivo de serem mais precisos na aplicação da medida. Esse tipo de avaliação permite que o tribunal tenha informações não só do ato infracional praticado, mas também do adolescente enquanto pessoa em desenvolvimento, com suas particularidades pessoais, familiares e acadêmicas. Sendo assim, aquele determinado adolescente, na frente do juiz na audiência de aplicação da medida, deixa de ser um adolescente qualquer que praticou um ato infracional e passa a ser um adolescente específico, com problemas específicos. Adicionalmente, não mais se trata de um crime praticado por um adolescente, mas de um adolescente que praticou um ato infracional; isto é, o foco é desviado do crime para a pessoa, deixandose de ver somente o crime e a punição e passando-se a olhar para o adolescente que porventura praticou aquele crime.

\section{ATUAÇÃO DO PSICÓlOGO}

$\mathrm{Na}$ cidade de London, na província de Ontário, essa avaliação é feita pelo Centre for Children and 
Families in the Justice System of the London Family Court Clinic, Inc. que também recebe adolescentes de outras cidades da região. Esse centro conta com pesquisadores renomados na literatura que atuam na University of Western Ontario como David Wolfe, Peter Jaffe, Allan Leschied, Naomi Rae-Grant, entre outros. A produção contínua do centro fez com que ele seja um ponto de referência no Canadá e no mundo. $\mathrm{O}$ centro também já organizou dois congressos internacionais, amplos, sobre os efeitos da exposição à violência em diferentes contextos.

No Brasil não é comum tal tipo de avaliação psicológica antes da aplicação da medida socioeducativa. Geralmente uma avaliação psicossocial é realizada no início do processo socioeducativo, após a aplicação da medida, por parte da equipe técnica de organizações responsáveis pela execução das medidas socioeducativas (Cortegoso et al., 2004).

O Centro recebe o encaminhamento do juiz e agenda uma primeira entrevista com o adolescente e seus pais. O trabalho do Centro é interdisciplinar e a entrevista de triagem (intake form) é realizada por um assistente social. Nessa entrevista, são obtidas informações sobre o adolescente, como: problemas na infância, relacionamento e dinâmica familiar, histórico de doenças e infrações à lei, grau de escolaridade, histórico escolar (classe especial, problemas na escola), uso de álcool e drogas. O adolescente assina um termo de consentimento autorizando o processo de avaliação e um termo autorizando o Centro a obter informações de outros órgãos, como escola, polícia, médicos e outras fontes de informações que forem relevantes. A mesma entrevista é feita com o adolescente e com os pais, a fim de se obter a informação de diferentes pontos de vista. É essencial que o adolescente assine o termo de consentimento, pois, sem autorização por escrito, nenhum profissional pode divulgar informações sobre o caso, nem para outros profissionais que estejam sob a égide do sigilo profissional. Qualquer cidadão canadense acima de 16 anos de idade tem suas informações pessoais garantidas por sigilo, sob a lei do sigilo (Privacy Act). Isso significa que qualquer profissional, inclusive $o$ psicólogo, é obrigado a manter sigilo de quaisquer informações, só podendo revelá-las a outros profissionais caso o cliente o autorize por escrito.

Em outra sessão, um psicólogo faz a aplicação de uma bateria de testes (Youth Self Report - Achenbach \& Edelbrock, 1984; Anger Screening Inventory Reynolds, 2001; Adolescent Mental Health Questionnaire - Landgraf, Abetz \& Ware, 1999; Adolescent Psychopathology Scale/Clinical Score
Report - Reynolds, 2001; e MACI - Millon Adolescent Clinical Inventory - Millon \& Davis, 1998), e também uma entrevista clínica com os pais do adolescente. Algumas entrevistas clínicas são feitas após a aplicação dos testes, com o objetivo de obter informações complementares mais precisas.

Adicionalmente, as informações prestadas pela escola, médicos, Conselho Tutelar (Children Aid Society) e outros, são analisadas em conjunto com as informações obtidas na avaliação, e monta-se um relatório no qual se explica como o jovem se apresenta psicossocialmente. O objetivo desse relatório é prover o tribunal de informações capazes de explicar possíveis razões que poderiam ter levado o adolescente a cometer um ato infracional, para assim poder definir eventuais estratégias de intervenção. $\mathrm{O}$ Centro também sugere a intervenção mais adequada ao caso.

Nessa avaliação interdisciplinar, os diversos profissionais que fazem parte da equipe do Centro compartilham as informações obtidas em cada caso e as analisam em conjunto, a fim de traçar um perfil psicossocial do adolescente, assim como os pontos importantes para uma intervenção. A equipe é formada por psicólogos clínicos, psicólogos jurídicos (criminalistas), assistentes sociais, educadores e psiquiatras. Nessa avaliação buscam-se os fatores de risco e de proteção envolvidos. Entende-se por fatores de risco condições ou variáveis associadas à alta probabilidade de ocorrência de resultados negativos ou indesejáveis, encontrando-se entre tais fatores os comportamentos que podem comprometer a saúde, o bem-estar ou desempenho social do indivíduo (Webster-Stratton, 1998). Já os fatores de proteção são aqueles que modificam ou alteram a resposta pessoal para algum risco ambiental que predisponha a resultados mal-adaptados (Rutter, 1979).

A literatura tem demonstrado que há uma convergência na descrição das características de jovens em conflito com a lei em diferentes culturas (Hagell \& Newburn, 1996; Hallahan \& Kauffman, 2000; Huizinga, Loeber, Thornberry \& Cothern, 2000; Kaplan, Sadock \& Grebb, 1997; Kauffman, 2001; Leschied, Andrews \& Hoge, 1993; Meichenbaum, 2001; Padovani, 2003; Patterson, Reid \& Dishion, 1992; Tremblay, Zhou, Gagnon, Vitaro \& Boileau, 1999). Entre as principais características estão: violação persistente de normas e regras sociais, comportamento desviante das práticas culturais vigentes, dificuldade para socializar, uso precoce de tabaco, de drogas e bebidas alcoólicas, história de comportamento antisocial, envolvimento em brigas, impulsividade, 
humor depressivo, tentativas de suicídio, ausência de sentimento de culpa, hostilidade, destruição de patrimônio público, institucionalização, incidentes de atear fogo, vandalismo, rejeição por parte de professores e colegas, envolvimento com pares desviantes, baixo rendimento acadêmico, fracasso e evasão escolar.

Geralmente a medida alternativa prevista em lei é considerada insuficiente. Acredita-se que a restrição de liberdade, por si só, seja incapaz de promover mudanças de comportamento. Quando um adolescente é levado a julgamento pela prática de um ato infracional, ele é responsabilizado, por alguma medida prevista no Youth Criminal Justice Act. O objetivo, além da "punição" pelo Estado, é que esse mesmo adolescente não volte a praticar ato infracional. Por essa razão, além da medida alternativa aplicada como forma de responsabilizar o adolescente pelo ato praticado, outros serviços são determinados para promover mudanças de comportamento. Segundo o Canadian Center for Justice Statistics (1998), no ano de 1997, em todo o país, 68\% dos adolescentes infratores receberam a medida de liberdade assistida (probation), 30\% a medida de prestação de serviços comunitários (community services), 20\% semiliberdade (open custody), 16\% internação (secure custody), 6\% multa (fine), e em 2\% houve arquivamento do processo por falta de evidências (absolute discharge).

Além dessas medidas que são decididas pelo tribunal, o juiz pode determinar outras, baseado no relatório do Centro, como um serviço de aconselhamento, atendimento clínico (psicológico e/ou psiquiátrico), sendo que o adolescente é obrigado a participar desses serviços concomitantemente ao cumprimento da medida. Por exemplo, se um jovem for acusado de crime sexual, além de receber, possivelmente, uma medida de liberdade assistida (a mais comum), poderá ser encaminhado para um grupo de jovens agressores sexuais em algum centro que ofereça esse serviço. Se existirem indícios de doença mental, além da medida, o adolescente poderá ser encaminhado a um hospital que disponha de unidade-dia para o atendimento ou participar do serviço clínico fornecido pelo centro. Na cidade de London, alguns casos diagnosticados como esquizofrenia são encaminhados para o Child and Parent Resource Institute, que é um hospital interligado ao Ministério da Criança e Serviços Juvenis (Ministry of Children \& Youth Services). Esse hospital também realiza pesquisas na área, em colaboração com a equipe do Centro e da University of Western Ontario.

O papel do psicólogo, nesses casos, não se restringe à avaliação. Segundo Cunningham (2002), a intervenção comumente realizada é a "terapia multissistêmica" (Multisystemic Therapy). Esse tipo de intervenção é intensivo e realizado na família de adolescentes em conflito com a lei. O trabalho envolve atendimento a toda a família do adolescente por um período de um a três meses, o qual ocorre na casa do cliente, em horários flexíveis, para atender a todos os membros da família. A intervenção leva em consideração as necessidades de cada membro da família, assim como seus valores, crenças e cultura. O objetivo dessa intervenção é identificar o problema apresentado dentro de contextos mais amplos, avaliando a relação do adolescente com sua família, com a comunidade em geral e com os serviços disponíveis, como escola, serviços de saúde e outros. A partir das relações entre esses sistemas, o terapeuta deve enfatizar as características positivas de cada sistema e usá-las como alavanca para melhorar as relações com os demais sistemas. A intervenção ocorre diariamente, endereçando problemas específicos e bemdefinidos, com vista ao empoderamento da família.

Entende-se por empoderamento de famílias, segundo Williams e Aiello (2004), o processo pelo qual famílias adquirem conhecimento, habilidades e recursos para terem um controle maior sobre suas vidas e melhorarem a qualidade de seu bem-estar. Isto é, em vez de se olhar para que tipo de ajuda as pessoas necessitam, deve-se olhar para as competências que já estão presentes no repertório e as novas oportunidades que podem surgir devido a novos repertórios (Singh, Curtis, Ellis, Nicholson, Villani \& Weschsler, 1995).

As escolas participam ativamente do processo de prevenção da violência, seja identificando casos de agressão dentro da escola e encaminhando esses casos para a polícia, seja fornecendo atividades que possam ser entendidas como preventivas, como um currículo voltado ao respeito à diversidade, atividades de cooperação e controle de raiva no ambiente escolar, tudo de acordo com uma política de governo da província de Ontário chamada Safe Schools Act - ato de segurança nas escolas (Heslop, Enright, Cunnigham, Hurley \& Stevens, 2006).

As escolas desempenham um papel importante na prevenção de comportamentos anti-sociais e infrações à lei (Gallo, 2006; Gallo \& Williams, 2005). Gallo e Williams (2005) apontaram que 
$60,2 \%$ dos adolescentes em conflito com a lei no Brasil abandonaram os estudos em função das dificuldades das escolas em manter tais alunos nas salas de aula. Verificou-se que todos os adolescentes atendidos pelo Centre for Children and Families in the Justice System freqüentavam a escola.

É consenso que os adolescentes em conflito com a lei buscam conseqüências imediatas para seus comportamentos (Patterson, Reid \& Dishion, 1992). Freqüentar um curso implica em lidar com conseqüências a longo prazo, além de envolver o comportamento de seguir regras, essencial para atividades acadêmicas (Patterson, DeBaryshe \& Ramsey, 1989; Rossi, 2001).

Kauffman (2001) aponta que um nível baixo de escolaridade atua como um fator de risco e, inversamente, um nível alto atua como fator de proteção. No estudo de Gallo e Williams (2005), os participantes que tinham a mais baixa escolaridade ( $1^{\mathrm{a}}$ a $4^{\mathrm{a}}$ séries) cumpriam, em sua maioria, uma medida socioeducativa severa (liberdade assistida), enquanto os participantes que tinham um nível educacional maior ( $5^{\mathrm{a}}$ a $8^{\mathrm{a}}$ série) cumpriam uma medida menos severa (prestação de serviços à comunidade).

Além disso, ainda no estudo de Gallo e Williams (2005), 67,3\% dos adolescentes que freqüentavam a escola não eram usuários de drogas, e entre os que não estudavam, a porcentagem de não-usuários caiu para 45,9\%. Esse dado é condizente com a alegação de pesquisadores de que freqüentar a escola é um fator de proteção contra o consumo de drogas (Kauffman, 2001; Rutter, 1979). O mesmo também é válido para o uso de armas, isto é, armas foram usadas primordialmente pelos participantes que não estavam no ensino formal (Freitas, 1997; Garmezy, 1985).

Considerando-se esses dados apresentados em estudos brasileiros, é possível a comparação com estudos canadenses. A legislação canadense exige a freqüência à escola de todos adolescentes até os 16 anos de idade. Se por acaso o adolescente decide abandonar os estudos, a família é punida por não respeitar essa lei. As formas mais comuns de punição são: perda de incentivos e benefícios governamentais, como auxílio à renda para famílias mais pobres, descontos em imposto de renda, incentivo governamental para micro e pequenas empresas. Além da perda desses benefícios, as famílias podem ser formalmente processadas pelo Conselho Tutelar (Children Aid Society), o que pode implicar em pagamento de multa e até a restrição judicial de direitos.

O maior problema enfrentado pelas escolas brasileiras ao lidarem com adolescentes em conflito com a lei são seus comportamentos disruptivos, que levam a escola a adotar medidas disciplinares coercitivas, as quais, por sua vez, facilitam a evasão escolar. $O$ professor brasileiro não recebe capacitação e incentivo para lidar com essa população. A falta de capacitação dos professores para atuar com alunos que apresentam problemas de comportamento, como jovens em conflito com a lei, e os baixos salários, que os desestimulam, são assuntos prementes.

As escolas canadenses têm uma série de programas de apoio educacional e de eeducação especial, que estão disponíveis na escola regular. Em horário regular da escola, os alunos freqüentam esses programas, de acordo com suas necessidades. O programa mais comum é o IEP (Individualized Education Plan), um plano individualizado, de acordo com as necessidades do aluno. A existência desses programas de apoio educacional é fruto do preparo que as escolas canadenses têm para lidar com alunos que apresentam problemas comportamentais em sala de aula. A legislação canadense determina que os professores desses programas tenham, no mínimo, mestrado em Educação Especial, capacitação que se reflete na qualidade do serviço prestado. Tudo isso só foi possível devido ao grande investimento do governo canadense na área de educação.

No Brasil, a intervenção se restringe, normalmente, àquela realizada pela equipe de execução das medidas socioeducativas, e geralmente não há outros serviços disponíveis. Depois da municipalização das medidas socioeducativas houve um esforço pioneiro em prover um atendimento diferenciado, no qual se levassem em conta as particularidades de cada adolescente, como observado no Programa de Medidas SocioEducativas em Meio Aberto e no Núcleo de Atendimento Integrado, ambos na cidade de São Carlos, SP.

Uma maior parceria entre o sistema judiciário e as universidades, especialmente no tocante à execução das medidas socioeducativas, como observado no Canadá, pode promover conhecimento específico cientificamente fundamentado. Além disso possibilitaria à universidade fazer pesquisas, assim como executar serviços de extensão complementares à medida socioeducativa, gerando um trabalho 
multidisciplinar, que poderia prevenir reincidências. Esse tipo de relacionamento, que tem como benefício uma intervenção cientificamente embasada, ainda é escasso no Brasil.

\section{REFERÊNCIAS}

Achenbach, T. M. \& Edelbrock, C. S. (1984). Psychopathology of childhood. Annual Review of Psychology, 35, 227-256.

Brasil (1990). Estatuto da Criança e do Adolescente. Juiz de Fora: Universidade Federal.

Canadian Centre for Justice Statistics (1998). A profile of youth justice in Canada. Ottawa: Minister of Industry Canada.

Canadian Centre for Justice Statistics (2000). Canadian crime statistics 1999. Ottawa: Minister of Industry Canada.

Cortegoso, A. L., Aroni, A., Gallo, A. E., Padovani, R. C., Macedo, I., Staniscia, A. C. M., Pereira, D. E. M., Santos, E. F., Silveira, G. M., Leugi, G. B., Santos, J. A., Gonçalves, T. N., Angelucci, T. C. \& Andrade, T. S. C. (2004). Sistematização de ações do NAI na implementação do Estatuto da Criança e do Adolescente. Relatório técnico de atividade curricular integrada de ensino, pesquisa e extensão. Departamento de Psicologia, Universidade Federal de São Carlos.

Cunningham, A. (2002). On step forward: Lessons learned from a randomized study of multisystemic therapy in Canada. Praxis - Research from the Centre for Children and Families in the Justice System. Retrieved in March, 2005, from http://www.lfcc.on.ca.

Freitas, M. G. (1997). Aspectos do desenvolvimento de um grupo de adolescentes albergados e suas implicações para uma intervenção psicoeducacional. Dissertação de Mestrado Publicada. Programa de Pós-Graduação em Educação. Universidade Estadual de Londrina, Londrina.

Gallo, A. E. (2006). Adolescentes em conflito com a lei: perfil e intervenção. Tese de Doutorado Publicada. Programa de PósGraduação em Educação Especial, Universidade Federal de São Carlos, São Carlos.

Gallo, A. E., \& Williams, L. C. A. (2005). Adolescentes em conflito com a lei: uma revisão dos fatores de risco para a conduta infracional. Psicologia: Teoria e Prática, 7(1), 81-95.

Garmezy, N. (1985). Stress resistant children: The search for protective factors. In J. Stevenson (Ed.), Recent research in developmental psychology. Oxford: Pergamon.

Hagell, A. \& Newburn, T. (1996). Family and social contexts of adolescent re-offenders. Journal of Adolescence, 19, 5-18.

Hallahan, D. P. \& Kauffman, J. M. (2000). Learners: Introduction to special education. Englewood Cliffs: Prentice-Hall.

Heslop, L., Enright, C., Cunningham, A., Hurley, P. \& Stevens, L. (2006). When teens hurt teens: Helping the victims of youthon-youth criminal violence. London (Canada): Centre for Children and Families in the Justice System e Victim Service Unit of London Police.

Huizinga, D., Loeber, R., Thornberry, D. \& Cothern, L. (2000). Co-occurrence of delinquency and other problem behavior. Juvenile Justice Bulletin, Retrieved in March, 2005, from http://www.ojjdp.ncjrs.org/ccd/index.html.
Kaplan, H. I., Sadock, B. I. \& Grebb, J. A. (1997). Compêndio de psiquiatria: ciências do comportamento e psiquiatria clínica. Porto Alegre: Artes Médicas.

Kauffman, J. M. (2001). Characteristics of emotional and behavioral disorders of children and youth. Upper Saddle River: Merrill Prentice Hall.

Landgraf, J. M., Abetz, I. \& Ware, J. E. (1999). The Adolescent Health Questionnaire: A user's manual. Boston: The Health Institute - New England Medical Center.

Leschied, A. W., Andrews, D. A. \& Hoge, R. D. (1993). A review of Ontario young offenders: Programs and literature that supports effective intervention. Retrieved in March, 2005, from http://www.lfcc.on.ca/risk1.htm.

Meichenbaum, D. (2001). Treatment of individuals with angercontrol problems and aggressive behaviors: A clinical handbook. Clearwater: Institute.

Meneghel, S. N., Giugliani, E. J. \& Falceto, O. (1998). Relações entre violência doméstica e agressividade na adolescência. Cadernos de Saúde Pública, 14(2), 327-335.

Millon, T. \& Davis, R. D. (1998). Ten subtypes of psychopathy. In T. Millon, E. Simonsen, M. Birket-Smith \& R. D. Davis (Eds.), Psychopathy: Antisocial, criminal and violent behavior. New York: Guilford.

Millon, T., Millon, C., Davis, R. \& Grossman, S. (1998). MACIMillon Adolescent Clinical Inventory. New York: Dicandrien.

Oliveira, M. B. \& Assis, S. G. (1999). Os adolescentes infratores do Rio de Janeiro e as instituições que os "ressocializam": a perpetuação do descaso. Cadernos de Saúde Pública, 15(4), 831-844.

Padovani, R. C. (2003). Resolução de problemas com adolescentes em conflito com a lei: uma proposta de intervenção. Dissertação de Mestrado Publicada, Programa de Pós-Graduação em Educação Especial, Universidade Federal de São Carlos, São Carlos.

Patterson, G. R., DeBaryshe, B. D. \& Ramsey, E. (1989). A developmental perspective on antisocial behavior. American Psychologist, 44(2), 115-136.

Patterson, G. R., Reid, J. B. \& Dishion, T. J. (1992). Antisocial boys. Eugene: Castalia.

Pereira, I. \& Mestriner, M. L. (1999). Liberdade assistida $e$ prestação de serviços à comunidade: medidas de inclusão social voltadas a adolescentes autores de ato infracional. São Paulo: IEE/PUC-SP; FEBEM-SP.

Reynolds, W. M. (2001). Adolescent adjustment screening inventory. New York: Harcourt Assessment.

Reynolds, W. M. (2001). Adolescent Psychopathology Scale: Clinical score report. Lutz: PAR Psychological Assessment Resources.

Rossi, T. M. F. (2001). Significações de professores sobre a escola: o caso de uma escola em um assentamento habitacional no Distrito Federal. Em A. Del Prette (Ed.), Psicologia escolar e educacional: saúde e qualidade de vida (p. 177-199). Campinas: Vozes.

Rutter, M. (1979). Protective factors in children's response to stress and disadvantage. In M. W. Kent \& J. E. Rolf (Eds.), Primary prevention of psychopathology: Social competence in children (p. 49-74). Hanover: New England University. 
Singh, N. N., Curtis, W. J., Ellis, C. R., Nicholson, M. W., Villani, T. M. \& Weschsler, H. A. (1995). Psychometric analysis of the family empowerment scale. Journal of Emotional and Behavioral Disorders, 3(2), 85-91.

Tremblay, R. E., Zhou, R. M., Gagnon, C., Vitaro, F. \& Boileau, H. (1999). Violent boys: Development and prevention. Correctional Services Canada. Retrieved in March, 2005, from http://www.correctionalservicecanada.com.

Webster-Stratton, C. (1998). Early intervention for families of preschool children with conduct problems. In M. J. Guralnick (Ed.), The effectiveness of early intervention (pp. 429-455). Baltimore: Paul H. Brookes.
Williams, L. C. A. \& Aiello, A. L. R. (2004). Empoderamento de famílias: o que vem a ser e como medir? Em E. G. Mendes, M. A. Almeida \& L. C. A. Williams (Orgs.), Temas em educação especial: avanços recentes (pp.197-202). São Carlos: EDUFSCar.

Endereço para correspondência: Alex Eduardo Gallo. Rua Oswaldo Cruz, 543, ap. 203, CEP 87020-200, Maringá-PR. E-mail: aedgallo@yahoo.com.br 